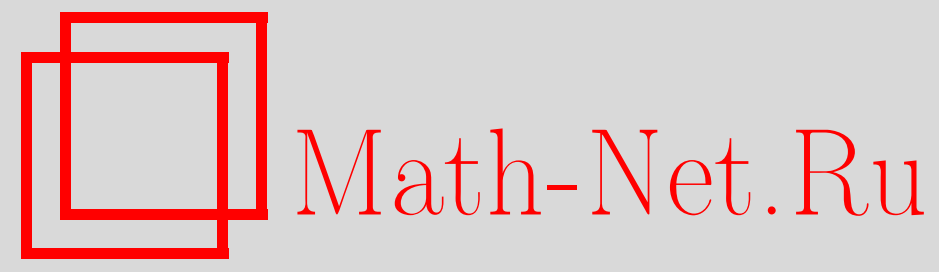

H. L. Hurd, A. Russek, Stepanov almost periodically correlated and almost periodically unitary processes, Теория вероятн. и ее примен., 1996, том 41, выпуск 3, 591-611

DOI: https://doi.org/10.4213/tvp3140

Использование Общероссийского математического портала Math-Net.Ru подразумевает, что вы прочитали и согласны с пользовательским соглашением http://www.mathnet.ru/rus/agreement

Параметры загрузки:

IP : 54.80 .97 .219

26 апреля 2023 г., $15: 31: 56$

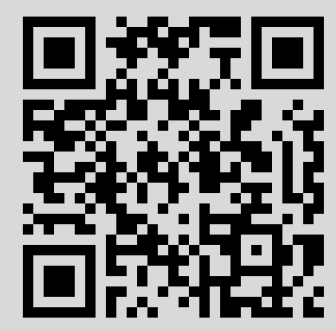




\title{
STEPANOV ALMOST PERIODICALLY CORRELATED AND ALMOST PERIODICALLY UNITARY PROCESSES ${ }^{1)}$
}

\begin{abstract}
Данная работа распространяет структуру и свойства почти периодически коррелированных (ППК) и почти периодически унитарных (ППУ) процессов, которые были определены в смысле Бохра, на более широкий класс процессов, для которых почти периодичность понимается в смысле Степанова. Эти процессы, в отличие от бохровских ППК и ППУ процессов, не являются с необходимостью непрерывными в среднем квадратическом, но сохраняют смысл почти периодичности. Например, процессы $f(t) X(t)$ или $X(t+f(t))$, полученные из стационарного в широком смысле процесса $X(t)$ в результате модуляций амплитуды или времени с помощью скалярной почти периодической (ПП) функции Степанова $f(t)$, не обязательно непрерывной, являются ППУ и ПІКК по Степанову. Основные результаты, касаюшиеся ППК и ІІПУ процессов, перенесены на новый класс процессов. Характеризация Гладышева ППК корреляционных функций распространена на ППК по Степанову процессы, и показано, что их коррелящионные функции полностью представляются рядом Фурье, имеющим счетное число функциональных коэффициентов, которые представляют собой преобразования Фурье комплексных мер. Установлено, что ППУ по Степанову процессы являются также ППК по Степанову и даются формулой $X(t)=U(t)[P(t)]$, где $\{U(t), t \in \mathbf{R}\}$ есть строго непрерывная группа унитарных операторов, а $P(t)$ есть векторнозначная ПП по Степанову функция. Как. и в случае ППУ по Бохру процессов, предыдущий факт приводит к представлениям $X(t)$, основанным на спектральной теории для унитарных операторов и для степановских почти периодических функций.
\end{abstract}

Ключевые слова и фразы: почти периодически коррелированные, почти периодически унитарные случайные процессы, почти периодические функции.

\section{Introduction}

Although discontinuous (in quadratic-mean) weakly stationary processes are considered pathological, there are simple examples of discontinuous second order nonstationary processes. Consider the process $X(t)=f(t) a(t)$,

*Harry L. Hurd Assoc., 309 Moss Run, Raleigh, N.C. 27614 U.S.A.

${ }^{* *}$ Instytut Matem. PAN, Abrahama 18, 81-825 Sopot, Poland.

1) Работа поддержана ONR, контракты N00014-89-C-0310 и N00014-C-92-0057. 
where $f(t)=f(t+T)$ is a periodic discontinuous scalar function and $a(t)$ is a quadratic-mean (q.m.) continuous weakly stationary process. In this case, $X(t)$ is periodically correlated $(\mathrm{PC})[4]$ in that $m(t)=\mathbf{E}\{X(t)\}=$ $\mathbf{E}\{X(t+T)\}$ and $R(s, t)=\mathbf{E}\{X(s) \overline{X(t)}\}=R(s+T, t+T)$; as our interest here is in the second order properties, we take $m(t) \equiv 0$. The periodicity of the correlation function $R(s, t)$ of a PC process is in a diagonal sense so that the transformation $s=t+\tau, t=t$ rotates the coordinates to put all the periodicity in the coordinate of local time $t$. That is, $B(t, \tau)=R(t+\tau, t)$ is periodic in the variable $t$ with period $T$. Thus the Fourier series,

$$
B(t, \tau)=\sum_{k} B_{k}(\tau) \exp \left[\frac{i 2 \pi k t}{T}\right]
$$

is suggested where $B_{k}(t)=(1 / T) \int_{0}^{T} B(t, r) \exp (-i 2 \pi k t / T) d t$, and the convergence of (1.1) depends on the properties of $B(\cdot, \tau)$. Gladyshev [4] gave necessary and sufficient conditions in terms of $\left\{B_{k}(\tau), k \in \mathbf{Z}\right\}$ for a continuous function $R(s, t)=R(s+T, t+T)$ to be non-negative definite. A similar result applies to a class of discontinuous correlation functions, including those arising from processes of the sort $X(t)=f(t) a(t)$ mentioned above [5].

In the following examples, $X(t)$ is to be considered as a curve or a mapping from $\mathbf{R}$ to the square integrable random variables, denoted $L_{2}(\Omega)$, of a probability space $(\Omega, \mathcal{F}, \mathbf{P})$. For two elements $X$ and $Y$ in $L_{2}(\Omega)$, we denote $\langle X, Y\rangle=\mathbf{E}\{X \bar{Y}\}$.

Every second order PC process $\{X(t), t \in \mathbf{R}\}$, discontinuous or not, has a natural imbedded unitary operator $U_{X}$ for which $X(t+T)=U_{X} X(t)$ for every $t \in \mathbf{R}[8]$. If $X(t)$ is q.m. continuous, it is then periodically unitary $(\mathrm{PU})$; that is, there is a strongly continuous group $\left\{U\left(t^{\prime}\right), t^{\prime} \in \mathbf{R}\right\}$ of unitary operators for which

$$
\left\|X\left(t+t^{\prime}\right)-U\left(t^{\prime}\right) X(t)\right\|_{2}=0 \quad \text { for every } t \text { and } n \text { when } \quad t^{\prime}=n T,
$$

where $\|\cdot\|_{2}$ is the $L_{2}(\Omega)$ norm. If $R(t, t)$ is Lebesgue integrable on $[0, T]$, then it is $L_{2}([0, T])$ periodically correlated $[8]$ in that

$$
\int_{0}^{T}\left\|X\left(t+t^{\prime}\right)-U\left(t^{\prime}\right) X(t)\right\|_{2}^{2} d t=0 \quad \text { when } \quad t^{\prime}=n T .
$$

Properties of the $L_{2}([0, T]) \mathrm{PU}$ processes are addressed in [8].

The same simple multiplicative example, $X(t)=f(t) a(t)$ with $f(t)$ uniformly almost periodic (UAP) in the sense of Bohr, is almost periodically correlated (APC) in the sense of Gladyshev [4]. That is, $m(t)$ is UAP and for every $s, t, R(s+\alpha, t+\alpha)$ is UAP in the variable $\alpha$. It follows that for every $\tau$, the function $R(t+\tau, t)$ is a UAP function with respect to the variable $t$ and so the correlation $R(t+\tau, t)$ has the Fourier series $\sum_{\lambda_{j} \in \Lambda_{\tau}} a\left(\lambda_{j}, \tau\right) \exp \left(\lambda_{j} t\right)$ 
where the coefficient functions are given by

$$
a\left(\lambda_{j}, \tau\right)=\lim _{A \rightarrow \infty} \frac{1}{2 A} \int_{-A}^{A} R(t+\tau, t) \exp \left(-i \lambda_{j} t\right) d t .
$$

For fixed $\tau$ the set $\Lambda_{\tau}$ is countable from the theory of UAP functions; for Gladyshev APC processes, it is also true that $\Lambda=\{\lambda: a(\lambda, \tau) \neq 0$ for some $\tau\}=\cup_{\tau} \Lambda_{\tau}$ is countable [7]. Gladyshev also gave necessary and sufficient conditions, in terms of the coefficient functions $a\left(\lambda_{j}, \tau\right)$, for $R(s, t)$ to be non-negative definite. The notion of periodically unitary processes defined by (1.2) motivates the almost periodically unitary (APU) processes [6]. These are the q.m. continuous second order processes $X(t)$ for which there exists a strongly continuous group $\left\{U\left(t^{\prime}\right), t^{\prime} \in \mathbf{R}\right\}$ of unitary operators such that for every $\varepsilon>0$ the set

$$
S(\varepsilon, X, U)=\left\{t^{\prime}: \sup _{t \in \mathbf{R}}\left\|X\left(t+t^{\prime}\right)-U\left(t^{\prime}\right) X(t)\right\|_{2}<\varepsilon\right\}
$$

is relatively dense (RD), or in more modern terms, has bounded gaps. A set $S$ is called RD if there exists a number $l>0$ such that every interval of length $l$ contains at least one point of $S$. Such an $l$ is called an inclusion length for $S$. The APU processes are precisely those processes given by $X(t)=\sum_{j} f_{j}(t) a_{j}(t)$ where $\left\{f_{j}(t), j \in \mathbf{Z}\right\}$ are UAP functions with $\sum_{j}\left|f_{j}(t)\right|^{2} \leqslant M$ and the convergence of $\sum_{j=1}^{n}\left|f_{j}(t)\right|^{2}$ is uniform in $t$; the processes $\left\{a_{j}(t), j \in \mathbf{Z}\right\}$ are jointly stationary and mutually orthogonal at each fixed $t,\left\langle a_{j}(t), a_{k}(t)\right\rangle=\delta_{j k}$. Equivalently, the APU processes are precisely those given by $X(t)=U(t)[P(t)]$ where $P(t)$ is a $L_{2}(\Omega)$-valued almost periodic function. Such a function might also be called an almost periodic process and $\left\{U\left(t^{\prime}\right), t^{\prime} \in \mathbf{R}\right\}$ is a group of unitary operators.

The same multiplicative example $X(t)=f(t) a(t)$ motivates the consideration of the class of processes obtained when $a(t)$ is weakly stationary (and q.m. continuous) but $f(t)$ is in the class $S_{p}$ of scalar Stepanov [9], [10] almost periodic functions; that is, $f(t)$ is locally $L_{p}: \int_{0}^{1}|f(t+u)|^{p} d u<\infty$ for every $t$, and for every $\varepsilon<0$ the set

$$
S(\varepsilon, f)=\left\{t^{\prime}: \sup _{t \in \mathbf{R}} \int_{t}^{t+1}\left|f\left(u+t^{\prime}\right)-f(u)\right|^{p} d u<\varepsilon\right\}
$$

is RD. We note that $S_{p}$ contains the periodic extensions of $L_{p}[0,1]$. In this paper we introduce the Stepanov APC and APU processes and give some basic structural results. In Section 2 we define the Stepanov APC processes and extend Gladyshev's characterization [4] of correlation functions of (q.m. continuous) APC processes to this case, for which the correlation need not be continuous. In Section 3 we define the Stepanov APU processes, give several characterizations of these processes and show that despite the lack of pointwise continuity of the map $X: \mathbf{R} \rightarrow L_{2}(\Omega)$, there is still a spectral theory analogous to the one developed in [6]. In particular the set $\Lambda=$ 
$\{\lambda: a(\lambda, \tau) \neq 0$ for some $\tau\}$ is again countable and the correlation $R(t+\tau, t)$ has the Fourier series $\sum_{\lambda_{j} \in \Lambda} a\left(\lambda_{j}, \tau\right) \exp \left(\lambda_{j} t\right)$, where the functions $a\left(\lambda_{j}, \cdot\right)$ are Fourier transforms of signed measures of finite total variation.

1.1. Stepanov almost periodic functions. In this section we summarize basic facts about Stepanov almost periodic functions. A primary fact due to Bochner [1] is that $f \in S_{p}$ if and only if the function $F: \mathbf{R} \rightarrow L_{p}[0,1]$ given by $F(t)=f(t+\cdot)$ is UAP. To be more precise, a function $f: \mathbf{R} \rightarrow \mathbf{B}$ (Banach space) is called almost periodic in the sense of Bohr if it is continuous and for every $\varepsilon>0$ the set

$$
S(\varepsilon, f)=\left\{t^{\prime}: \sup _{t}\left\|f\left(t+t^{\prime}\right)-f(t)\right\|_{\mathbf{B}}<\varepsilon\right\}
$$

is relatively dense (RD). Every $t^{\prime}$ in $S(\varepsilon, f)$ is called an $\varepsilon$-almost period of $f$.

Let $L_{p}(I, \mathrm{~B})$ denote the space of Lebesgue-Bochner measurable $L_{p}$ integrable $\mathrm{B}$-valued functions on $I=[0,1]$ with the usual norm

$$
\|\| F \|_{p}=\left(\int_{0}^{1}\|F(u)\|_{\mathrm{B}}^{p} d u\right)^{1 / p}<\infty
$$

A Lebesgue-Bochner measurable $\mathrm{B}$-valued function is also called strongly measurable $([12$, p. 130$])$. A strongly measurable $L_{p}$-integrable $\mathbf{B}$-valued function $F$ is Bochner- $L_{p}$ ([12, p. 133]); we shall say that $F \in L_{\mathbf{B}}^{p}$. A map $f: \mathbf{R} \rightarrow \mathbf{B}$ will be called locally $L_{\mathbf{B}}^{p}$ if it is locally strongly measurable and $\int_{0}^{1}\|f(t+u)\|_{\mathbf{B}}^{p} d u<\infty$ for every $t$. If $f$ is locally $L_{\mathbf{B}}^{p}$ and we define $F: \mathbf{R} \rightarrow L_{p}(I, \mathbf{B})$ by $F(t)=f(t+\cdot)$, then the approximations resulting from Bochner $-L_{p}$ integrability imply that $F$ is continuous at every $t$.

A locally $L_{\mathrm{B}}^{p}$ function $f$ is called Stepanov $S_{p}(\mathrm{~B})$ almost periodic or $S_{p}(\mathrm{~B})$ (see [9]) if for every $\varepsilon>0$ the set

$$
S_{p}(\varepsilon, f)=\left\{t^{\prime}: \sup _{t} \int_{t}^{t+1}\left\|f\left(u+t^{\prime}\right)-f(u)\right\|_{\mathbf{B}}^{p} d u<\varepsilon\right\}
$$

is $\mathrm{RD}$. Since the continuity of $F$ is obtained a-forteriori for every such $f$, it is easy to see that $f$ is in $S_{p}(\mathrm{~B})$ if and only if the corresponding $L_{p}(I, \mathrm{~B})$ valued function $F(t)=f(t+\cdot)$ is almost periodic in the sense of Bohr, i.e., it is continuous and for every $\varepsilon>0$ the set

$$
S(\varepsilon, F)=\left\{t^{\prime}: \sup _{t}\left\|\mid F\left(t+t^{\prime}\right)-F(t)\right\| \|_{p}<\varepsilon\right\}
$$

is RD [1]. The spaces of scalar valued Stepanov AP functions (i.e., $S_{p}(\mathbf{R})$ or $\left.S_{p}(\mathbf{C})\right)$ will be denoted by $S_{p}$, or $S_{p}$ AP.

For any $S_{p}$ function $f$ the mean value

$$
M_{t}\{f\}:=\lim _{T \rightarrow \infty} \frac{1}{2 T} \int_{-T+c}^{T+c} f(t) d t
$$


exists uniformly with respect to the parameter $c$. Further, it is clear that

$$
\left\|M_{t}\{f\}\right\|_{\mathbf{B}}^{p} \leqslant \sup _{t} \int_{0}^{1}\|f(t+s)\|_{\mathbf{B}}^{p} d s \leqslant \sup _{t}\|f(t)\|_{\mathbf{B}}^{p} .
$$

If $f$ is any $S_{p}$ almost periodic function, also is $f(t) \exp (-i \lambda t)$ and the Fourier coefficients

$$
a(\lambda)=M_{t}\{f(t) \exp (-i \lambda t)\}
$$

exist for every $\lambda$. The set $\Lambda=\{\lambda: a(\lambda) \neq 0\}$ is countable and the coefficients $a(\lambda)$ (for $\lambda \in \Lambda$ ) determine $f$ uniquely up to equality almost everywhere, i.e., if for every $\lambda$

$$
M_{t}\{f(t) \exp (-i \lambda t)\}=M_{t}\{g(t) \exp (-i \lambda t)\}
$$

then $\|f(t)-g(t)\|_{\mathrm{B}}^{p} \longrightarrow 0$ almost everywhere with respect to the Lebesgue measure on $\mathbf{R}$.

\section{Stepanov almost periodically correlated processes}

A complex valued second order stochastic process $X=X(t), t \in \mathbf{R}$, with correlation $R(s, t)=\mathbf{E}\{X(s) \bar{X}(t)\}$ is called $S_{p}$ almost periodically correlated $\left(S_{p} \mathrm{APC}\right)$ if for every $\tau$ the function

$$
B(t, \tau)=R(t+\tau, t)
$$

is $S_{p} \mathrm{AP}$.

For such $R(s, t)$ we define

$$
\begin{aligned}
a(\lambda, \tau) & =M_{t}\{B(t, \tau) \exp (-i \lambda t)\} \\
& =\lim _{T \rightarrow \infty} \int_{-T+c}^{T+c} R(t+\tau, t) \exp (-i \lambda t) d t .
\end{aligned}
$$

The limit does not depend on $c$ by (1.8).

Gladyshev [4] gives a necessary and sufficient condition for a continuous function $R(s, t)$, to be a correlation of an APC process. The necessity extends immediately to the $S_{p}$ APC case; in fact the same proof yields even a slightly more general theorem.

Proposition 2.1. Let $R(s, t), t, s \in \mathbf{R}$, be the correlation of an $S_{p} \mathrm{APC}$ process. Then for every real $t_{1}, \ldots, t_{n}, \mu_{1}, \ldots, \mu_{n}$ and complex $z_{1}, \ldots, z_{n}$ we have

$$
\sum_{k, l} z_{k} \bar{z}_{l} a\left(\mu_{l}-\mu_{k}, t_{k}-t_{l}\right) \exp \left[i \mu_{k}\left(t_{k}-t_{l}\right)\right] \geqslant 0 .
$$
pleteness.

P r o of. The proof is identical to Gladyshev's; we include it for com-

First for every $n$ and collections $\left\{t_{1}, \ldots, t_{n}\right\},\left\{w_{1}, \ldots, w_{n}\right\}$ we have

$$
\sum_{k, l} R\left(t_{k}+u, t_{l}+u\right) w_{k} \bar{w}_{l} \geqslant 0
$$


Then we note from (2.1) that

$$
\lim _{T \rightarrow \infty} \frac{1}{2 T} \int_{-T}^{T} R(s+u, t+u) \exp (-i \lambda u) d u=a(\lambda, s-t) \exp (i \lambda t)
$$

Substituting $\dot{w_{k}}=\exp \left(i \mu_{k}\left(u+t_{k}\right)\right) z_{k}$ and integrating with respect to $u$, we get

$$
\sum_{k, l} z_{k} \bar{z}_{l} \frac{1}{2 T} \int_{-T}^{T} R\left(t_{k}+u, t_{l}-u\right) \exp \left[-i\left(\mu_{l}-\mu_{k}\right) u\right] d u \exp \left[i\left(\mu_{k} t_{k}-\mu_{l} t_{l}\right)\right] \geqslant 0 .
$$

Now using (2.3) and after obvious calculations we conclude (2.2).

We now address the extension of the sufficiency of Gladyshev's theorem to the Stepanov APC case. We first note that the coefficients $a(\lambda, \tau)$ determine $R(t+\tau, t)$ only up to equality almost everywhere, thus a condition like (2.2) will not imply that $R$ is positive definite but at most that it is equal to a positive definite function almost everywhere in a sense to be made precise subsequently. Moreover, Gladyshev's proof requires that not only every $\Lambda_{\tau}$ but the union $\Lambda=\cap_{\tau} \Lambda_{\tau}$ be countable. Gladyshev does not address this issue but his proof can be augmented as follows: the uniform joint continuity of $R(t, s)$ implies that for each fixed $\lambda$ the function $\tau \rightarrow a(\lambda, \tau)$ is continuous; indeed

$$
\begin{aligned}
|a(\lambda, \tau+h)-a(\lambda, \tau)| & \leqslant M_{t}\{|R(t+\tau+h, t)-R(t+\tau, t)|\} \\
& \leqslant \sup _{t}|R(t+\tau+h, t)-R(t+\tau, t)|<\varepsilon
\end{aligned}
$$

for $|h|$ sufficiently small. Now continuity of $a(\lambda, \cdot)$ implies that if $a(\lambda, \tau)=0$ for all rational $\tau$ then $a(\lambda, \tau)=0$ for all $\tau \in \mathbf{R}$. It follows that

$$
\Lambda=\bigcup_{\tau \in \mathbf{R}} \Lambda_{\tau} \subset \bigcup_{\tau \text { rational }} \Lambda_{\tau}
$$

which implies that $\Lambda$ is at most countable.

The above reasoning may be used under the weaker assumption that $\tau \rightarrow B(\cdot, \tau)$ is continuous with respect to the $S_{p}$-norm; for then,

$$
M_{t}\{|R(t+\tau+h, t)-R(t+\tau, t)|\}^{p} \leqslant \sup _{t} \int_{t}^{t+1}|B(u, \tau+h)-B(u, \tau)|^{p} d u<\varepsilon
$$

for $|h|$ sufficiently small, and hence we can conclude that $\Lambda$ is countable. It also turns out that this continuity assumption is enough to extend Gladyshev's theorem to the $S_{p}$ case.

Theorem 2.1. Suppose $B(\cdot, \tau)=R(\cdot+\tau, \cdot)$ is $S_{p}$ almost periodic and $\tau \rightarrow B(\cdot, \tau)$ is a continuous function from $\mathbf{R}$ to $S_{p}$. Then if condition (2.2) is satisfied, there exist a positive definite function. $\widetilde{R}(t, s)$ and a set $M \subset \mathbf{R}$ of Lebesgue measure zero such that $R(t, s)=\widetilde{R}(t, s)$ for $(t, s) \notin M \times M$. 
$\mathrm{P} \mathrm{r}$ o of. The remark before the theorem shows that there exists one countable set $\Lambda=\left\{\lambda_{1}, \lambda_{2}, \ldots\right\}$ such that $a(\lambda, \tau)=0$ for $\lambda \notin \Lambda$ and $\tau \in \mathbf{R}$ so we can form the Bochner-Fejer approximating polynomials as in Gladyshev's theorem. Namely, let $\beta_{1}, \beta_{2}, \ldots$ be a rational basis of $\Lambda$, i.e., a subset of $\Lambda$ such that for every $\lambda \in \Lambda$ there exists a sequence of rational numbers $r_{1}, r_{2}, \ldots, r_{n}$ such that $\lambda=\sum_{j=1}^{n} r_{j} \beta_{j}$ and $\beta_{1}, \beta_{2}, \ldots$ are linearly independent over the field of rational numbers. Such a basis can always be selected from elements of $\Lambda$ ([9, p. 26]). The Bochner-Fejer polynomials are defined as follows $([9$, p. 27$])$ :

$$
R_{m}(u, v)=\frac{1}{(m !)^{2 m}} \sum_{v_{1}=0}^{(m !)^{2}-1} \cdots \sum_{v_{1}=0}^{(m !)^{2}-1} \sigma_{v_{1} \cdots v_{m}}(u, v)
$$

where

$$
\begin{aligned}
\sigma_{v_{1} \cdots v_{m}}(u, v)= & \sum_{k_{1}=-v_{1}}^{v_{1}} \ldots \sum_{k_{m}=-v_{m}}^{v_{m}} \exp \left\{i\left(\frac{v_{1}}{m !} \beta_{1}+\cdots+\frac{v_{m}}{m !} \beta_{m}\right) v\right\} \\
& \times a\left(\frac{v_{1}}{m !} \beta_{1}+\cdots+\frac{v_{m}}{m !} \beta_{m}, u-v\right) .
\end{aligned}
$$

Using the formula

$$
\sum_{v=0}^{m} \sum_{k=-v}^{v} a_{k}=\sum_{k, j=0}^{m} a_{k-j}
$$

we can rewrite the formula for $R_{m}$ as

$$
\begin{aligned}
R_{m}(u, v)= & \sum_{k_{1}, \ldots, k_{m}} \sum_{j_{1}, \ldots, j_{m}} a\left(\mu_{k_{1} \cdots k_{m}}-\mu_{j_{1} \cdots j_{m}}, u-v\right) \\
& \times \exp \left\{i\left(\mu_{k_{1} \cdots k_{m}}-\mu_{j_{1} \cdots j_{m}}\right) v\right\},
\end{aligned}
$$

where we have denoted

$$
\mu_{k_{1} \cdots k_{m}}=\frac{k_{1}}{m !} \beta_{1}+\cdots+\frac{k_{m}}{m !} \beta_{m}
$$

and the sum is taken over all $k_{i}, j_{i}$ such that $0 \leqslant k_{i}, j_{i} \leqslant(m !)^{2}-1$. Now for any $t_{1}, \ldots, t_{n}, z_{1}, \ldots, z_{n}$ after multiplying and dividing by $\exp \left\{i \mu_{j_{1} \cdots j_{m}}\left(t_{k}-\right.\right.$ $\left.t_{l}\right)$ \} we get

$$
\begin{aligned}
\sum_{k, l} R_{m}\left(t_{k}, t_{l}\right) z_{k} \bar{z}_{l}= & \sum_{k_{1}, \ldots, k_{m}} \sum_{j_{1}, \ldots, j_{m}} \sum_{k, l} a\left(\mu_{k_{1} \cdots k_{m}}-\mu_{j_{1} \cdots j_{m}}, t_{k}-t_{l}\right) \\
& \times \exp \left\{i \mu_{j_{1} \cdots j_{m}}\left(t_{k}-t_{l}\right)\right\} z_{k, j_{1} \cdots j_{m}} \bar{z}_{l, k_{1} \cdots k_{m}}
\end{aligned}
$$

where

$$
z_{k, j_{1} \cdots j_{m}}=\exp \left\{-i \mu_{j_{1} \cdots j_{m}} t_{k}\right\} z_{k}, \quad z_{l, k_{1} \cdots k_{m}}=\exp \left\{-i \mu_{k_{1} \cdots k_{m}} t_{l}\right\} z_{l} .
$$


The above expression is of the form (2.2) with $t_{k}, \mu_{k}, a_{k}, 1 \leqslant k \leqslant n$, replaced by multi-index sequences $t_{k, k_{1} \cdots k_{m}}, \mu_{k, k_{1} \cdots k_{m}}, z_{k, k_{1} \cdots k_{m}}$ where $t_{k, k_{1} \cdots k_{m}}=t_{k}$, $\mu_{k_{1} \ldots k_{m}}$ is given by $\left(2.3^{\prime}\right)$ and $z_{k, k_{1} \cdots k_{m}}$ is as above. Now condition (2.2) implies

$$
\sum_{k, l} R_{m}\left(t_{k}, t_{l}\right) z_{k} \bar{z}_{l} \geqslant 0
$$

which, in order to apply the approximation theorem, we rewrite as

$$
\sum B_{m}\left(t_{l}, t_{k}-t_{l}\right) z_{k} \bar{z}_{l} \geqslant 0 .
$$

For every $\tau, B_{m}(t, \tau)=R_{m}(t+\tau, t)$ is the Bochner-Fejer polynomial $\left(\left[9\right.\right.$, p. 27]) corresponding to the AP function $B(\cdot, \tau)$, thus $B_{m}(\cdot, \tau)$ converges to $B(\cdot, \tau)$ in the Stepanov norm $([9$, p. 30$])$, i.e.,

$$
\sup _{t} \int_{t}^{t+1}\left|R_{m}(u+\tau, u)-R(u+\tau, u)\right|^{p} d u \longrightarrow 0
$$

as $m \rightarrow \infty$.

Continuity of $\tau \longrightarrow B(\cdot, \tau) \in S_{p} \mathrm{AP}$ implies that for every $N$, the set of functions

$$
A_{N}=\{B(\cdot, \tau), \tau \in[-N, N]\}
$$

being an image of a compact set $[-N, N]$ is compact in $S_{p}$. By Lyusternik's theorem ([9, p. 7]) applied to $X=S_{p}$ this set of $S_{p}$ AP functions satisfies the following conditions:

(i) For every $t_{0} \in \mathbf{R}$, the set of $L_{p}(0,1)$-valued functions:

$$
E_{t_{0}, N}=E\left\{g \in L_{p}(0,1) ; g(u)=B\left(t_{0}+u, \tau\right), \tau \in[-N, N]\right\}
$$

is compact in $L_{p}(0,1)$.

(ii) Functions in $A_{N}$ are equicontinuous, i.e., for every $\varepsilon>0$ there exists a $\delta>0$ depending only on $N$ such that $\left|t^{\prime}-t^{\prime \prime}\right|<\delta$ implies

$$
\left\|B\left(t^{\prime}, \tau\right)-B\left(t^{\prime \prime}, \tau\right)\right\|_{S_{p}}<\varepsilon
$$

for every $\tau$ in $[-N, N]$.

(iii) $A_{N}$ is equi-almost periodic in the sense that for every $\varepsilon>0$ there is an $l=l_{\varepsilon}(N)$, depending on $N$ but not on $\tau \in[-N, N]$ such that every interval $(n, n+l) \subset \mathbf{R}$ contains a common $\varepsilon$-almost period for all $B(\cdot, \tau)$, $\tau \in[-N, N]$.

The proof of the approximation theorem $([9$, p. 29]) shows that conditions (i)-(iii) imply that $B_{m}(\cdot, \tau)$ converges to $B(\cdot, \tau)$ in $S_{p}$, uniformly in $\tau \in[-N, N]$; that is, for every $\varepsilon>0$ there exists $m_{0}=m_{0}(\varepsilon, N)$ such that for $m>m_{0}$ and $\tau \in[-N, N]$

$$
\sup _{t} \int_{t}^{t+1}\left|B_{m}(u, \tau)-B(u, \tau)\right|^{p} d u<\varepsilon
$$


From this it follows that for every $N, B_{m}(\cdot, \tau)$ converges in $L_{p}(-N, N)$ uniformly in $\tau \in[-N, N]$ and, hence, it converges in measure also uniformly in $\tau$, i.e., for every $\varepsilon_{1}, \varepsilon_{2}$ there exists $m>m_{0}\left(\varepsilon_{1}, \varepsilon_{2}, N\right)$ such that $n, m>m_{0}$ implies

$$
\operatorname{Leb}\left\{t:\left|B_{m}(t, \tau)-B_{n}(t, \tau)\right|>\varepsilon_{1}\right\}<\varepsilon_{2}
$$

for all $\tau \in[-N, N]$. Now applying a standard argument on existence of a subsequence converging almost surely (e.g., $\left[6\right.$, p. 59]), we can find $m_{n}$, depending only on $N$, and a set $M_{N} \subset \mathbf{R}$ of Lebesgue measure zero such that for every $t \notin M_{N}$ and $\tau \in[-N, N]$ we have

$$
\lim _{n \rightarrow \infty} B_{m_{n}}(t, \tau)=B(t, \tau)
$$

It follows from the above that there exists a subsequence $m_{\nu}$ such that unless $t \in M=\cup_{N} M_{N}, B_{m_{\nu}}(t, \tau)$ converges to $B(t, \tau)$. Now we have for any $t_{1}, \ldots, t_{n} \notin M$

$$
\sum_{k, l} B_{m_{\nu}}\left(t_{l}, t_{k}-t_{l}\right) z_{k} \bar{z}_{l}=\sum_{k, l} R_{m_{\nu}}\left(t_{k}, t_{l}\right) z_{k} \bar{z}_{l} \geqslant 0
$$

converges, as $\nu \rightarrow \infty$ to

$$
\sum_{k, l} B\left(t_{l}, t_{k}-t_{l}\right) z_{k} \bar{z}_{l}=\sum_{k, l} R\left(t_{k}, t_{l}\right) z_{k} \bar{z}_{l} \geqslant 0 .
$$

Setting $\widetilde{R}(t, s)=R(t, s)$ for $(t, s) \in(\mathbf{R} \backslash M) \times(\mathbf{R} \backslash M)$ and $\widetilde{R}(t, s)=0$ elsewhere concludes the proof of the theorem.

- $\mathrm{R}$ e $\mathrm{m}$ a $\mathrm{r} \mathrm{k}$. The difficulty in the last part of the proof of Theorem 2.1 is that we are trying to infer something about $R(t, s)=B(s, t-s)$ using convergence of $B_{m}(t, \tau)=R_{m}(t+\tau, t)$. If $R$ is jointly uniformly continuous as in Gladyshev's case, then $R_{m}(t, s)=B_{m}(s, t-s)$ converges to $B(s, t-s)=$ $R(t, s)$ for every $\tau=t-s$ and every $s$, hence, for every $t$ and $s$. In the present situation we only have, for every $\tau=t-s$, almost everywhere convergence with respect to $s$ in a set of Lebesgue measure zero, say $M_{\tau}$, and unless we can combine $\cup_{\tau} M_{\tau}$ into one set of measure zero we can not take a limit in (2.5) to get (2.6).

The same continuity that was instrumental in Theorem 2.1 permits us to conclude the following.

Theorem 2.2. Suppose $B(\cdot, \tau)=R(\cdot+\tau, \cdot)$ is $S_{p}$ almost periodic and $\tau \rightarrow B(\cdot, \tau)$ is a continuous function from $\mathbf{R}$ to $S_{p}$. Then for every $\lambda \in \mathbf{R}$ there exists a signed measure $\mu_{\lambda}$ of finite total variation for which

$$
a(\lambda, \tau)=\int_{-\infty}^{\infty} \exp (i \gamma \tau) \mu_{\lambda}(d \gamma)
$$

$\mathrm{R}$ e $\mathrm{m}$ a $\mathrm{r} \mathrm{k}$. Under the same hypotheses, $\mu_{\lambda}$ will be non-null for only a countable set of $\lambda$. 
P r o of. By remarks preceding Theorem 2.1, the function $\tau \rightarrow a(\lambda, \tau)$ is continuous for each $\lambda$ and since $a(0, \cdot)$ may be shown to be positive definite (see [7]), there exists a finite measure $\mu_{0}$ such that

$$
a(0, \tau)=\int_{-\infty}^{\infty} \exp (i \gamma \tau) \mu_{0}(d \gamma)
$$

Now continuity of $a(\lambda, \cdot)$ and Proposition 3 of [7] show that (2.7) holds for every $\lambda$ where $\mu_{\lambda}$ is a signed measure of finite total variation bounded by that of $\mu_{0}$, i.e.,

$$
\int_{-\infty}^{\infty}\left|\mu_{\lambda}(d \gamma)\right| \leqslant a(0,0)=\int_{-\infty}^{\infty} \mu_{0}(d \gamma)
$$

\section{Stepanov almost periodically unitary processes}

In this section, the Banach space $B$ of Section 1.1 is specialized to be $L_{2}(\Omega)$. For a second order stochastic process $X=\{X(t), t \in \mathbf{R}\}$ we denote by $H=H_{X}$ the $L_{2}(\Omega)$-closure of $\operatorname{span}\{X(t), t \in \mathbf{R}\} . H_{X}$ is a Hilbert space with the norm \|\|$_{2}=\|\|_{L_{2}(\Omega)}$. We consider processes $X$ that satisfy the following conditions:

Condition A: $X$ is locally strongly measurable,

Condition B: $\int_{t}^{t+1}\|X(u)\|_{2}^{p} d u<\infty$ for every $t$.

Conditions $\mathrm{A}$ and $\mathrm{B}$ together imply $X$ is locally $L_{2}^{p}([12, \mathrm{p} .133])$ and $X(t+\cdot)$ as a map $\mathbf{R} \rightarrow L_{p}\left(I, L_{2}(\Omega)\right)$ is continuous.

D e f i n i t i o $n$ 3.1. A second order stochastic process $X: \mathbf{R} \rightarrow L_{2}(\Omega)$ is called $S_{p}$ almost periodically unitary $\left(S_{p} \mathrm{APU}\right)$ if it satisfies conditions A and $\mathrm{B}$ and there exists a strongly continuous group $U\left(t^{\prime}\right), t^{\prime} \in \mathbf{R}$, of unitary operators on $H_{X}$ such that for every $\varepsilon>0$ the set

$$
S_{p}(\varepsilon, X, U)=\left\{t^{\prime}: \sup _{t} \int_{t}^{t+1}\left\|X\left(u+t^{\prime}\right)-U\left(t^{\prime}\right) X(u)\right\|_{2}^{p} d u<\varepsilon\right\}
$$

is $\mathrm{RD}$.

We now give some examples and describe basic properties of $S_{p} \mathrm{APU}$ processes. In each of the examples we must show that $X$ satisfies conditions $\mathrm{A}$ and $\mathrm{B}$ and there exists a strongly continuous group of unitary operators satisfying the preceding definition. To show $X$ satisfies condition A (locally strongly measurable) it suffices by a theorem of Pettis $([12$, p. 130]) to show

Condition $\mathbf{A}_{1}: X(t)$ is locally weakly measurable; that is, $\langle X(t), Z\rangle$ is a Borel measurable function of $t$ for every $Z \in H_{X}$;

Condition $\mathrm{A}_{2}: X(t)$ is almost separably valued: there is a Borel measurable set $E$ with $m(E)=0$ and the range of $X(t)$ for $t \notin E$ is separable.

$\mathrm{Ex}$ a $\mathrm{m} \mathrm{ple} 1$. First, an $L_{2}(\Omega)$-continuous wide sense stationary pro- 
cess is $S_{p}$ APU for $p>0$ with $U\left(t^{\prime}\right)$ a shift group defined by

$$
U\left(t^{\prime}\right)\left(\sum_{j=1}^{n} a_{j} X\left(t_{j}\right)\right)=\sum_{j=1}^{n} a_{j} X\left(t_{j}+t^{\prime}\right)
$$

as it follows that $\left\|X\left(u+t^{\prime}\right)-U\left(t^{\prime}\right) X(u)\right\|_{2}^{p}=0$ for every $t^{\prime}$ and $u$, so $S_{p}(\varepsilon, X, U)=\mathbf{R}$. The weak measurability comes from the fact that $\langle X(t), Z\rangle$ is continuous and therefore measurable function of $t$ for every $Z \in H_{X}$. Further, the continuity of $X$ implies the required separability. Finally, $\int_{t}^{t+1}\|X(u)\|_{2}^{p} d u<\infty$ for every $t$ because $\|X(u)\|_{2}^{p}=\|X(0)\|_{2}^{p}$ from stationarity.

Exa m ple 2. Every uniformly APU process is $S_{p}$ APU with any $p>0$. The weak measurability and separability come from the continuity of $X$ as in the stationary case. Further, $\int_{t}^{t+1}\|X(u)\|_{2}^{p} d u<\infty$ for every $t$ because $\|X(u)\|_{2}^{p}$ is continuous and bounded [6]. Finally, the fact that

$$
S(\varepsilon, X, U)=\left\{t^{\prime}: \sup _{t}\left\|X\left(t+t^{\prime}\right)-U\left(t^{\prime}\right) X(t)\right\|_{2}<\varepsilon\right\}
$$

is RD and $\left\|X\left(t+t^{\prime}\right)-U\left(t^{\prime}\right) X(t)\right\|_{2}$ is a continuous function of $t$ implies $S_{p}(\varepsilon, X, U)$ is RD. Thus in particular a trigonometric polynomial with jointly stationary coefficients, i.e., a process of the form

$$
X(t)=\sum_{j=1}^{n} a_{j}(t) \exp \left(i \lambda_{j} t\right)
$$

where $\left\{a_{j}(t), j=1, \ldots, n\right\}$ is a set of jointly wide sense stationary processes, is uniformly APU and hence $S_{p} \mathrm{APU}$.

E x a m pl e 3. If $\left\{a_{j}(t), j=1, \ldots, n\right\}$ are instantaneously orthogonal and q.m. continuous jointly wide sense stationary, and $\left\{f_{j}(t), j=1, \ldots, n\right\}$ are scalar $S_{p}$ almost periodic functions, then

$$
X(t)=\sum_{j=1}^{n} f_{j}(t) a_{j}(t)
$$

is $S_{p}$ APU. We shall make the argument only for $n=1$. The weak measurability comes from the fact that $\langle f(t) a(t), Z\rangle=f(t)\langle a(t), Z\rangle$ and the two factors are measurable. The process $f(t) a(t)$ is separably valued because $a(t)$ is separably valued and the scalar multiplier $f(t)$ can only eliminate vectors from the range of $a(t)$. Next, $\int_{t}^{t+1}\|f(u) a(u)\|_{2}^{p} d u=\|a(0)\|_{2}^{p} \int_{t}^{t+1}|f(u)|^{p} d u<\infty$ for every $t$ because $f(u)$ is $S_{p}$. Choosing the group $\left\{U\left(t^{\prime}\right), t^{\prime} \in \mathbf{R}\right\}$ to be the shift group for the stationary process $a(t)$, then $\left\|X\left(u+t^{\prime}\right)-U\left(t^{\prime}\right) X(u)\right\|_{2}^{p}=$ $\left|f\left(u+t^{\prime}\right)-f(u)\right|^{p}\left\|a\left(u+t^{\prime}\right)\right\|_{2}^{p}$ implies that $S_{p}(\varepsilon, X, U)$ is RD. This shows that amplitude scale modulations of wide sense stationary by scalar $S_{p}$ functions produces $S_{p} \mathrm{APU}$ processes.

$\mathrm{E} \times \mathrm{a} \mathrm{m} \mathrm{p} \mathrm{le} \mathrm{4.} \mathrm{The} \mathrm{next} \mathrm{class} \mathrm{of} \mathrm{examples} \mathrm{(time} \mathrm{scale} \mathrm{modulation)} \mathrm{is}$ contained in the following proposition. 
Proposition 3.1. Let $X$ be a mean square continuous wide sense stationary stochastic process and let $f$ be a scalar $S_{p}$ function. Then the process

$$
Y(t)=X(t+f(t))
$$

is $S_{p} \mathrm{APU}$.

$\mathrm{P}$ r o of. In this example we are required to replace $X(t)$ with a jointly measurable modification $\widetilde{X}(t)$ (this means $\widetilde{X}(t, \omega)=X(t, \omega)$ almost surely for each $t$ ) in order to obtain the weak measurability. For then, since $f(t)$ is measurable,

$$
\langle\tilde{X}(t+f(t)), Z\rangle=\int_{\Omega} \tilde{X}(t+f(t), \omega) \overline{Z(\omega)} d \mathbf{P}
$$

is a measurable function of $t$ for $Z \in H(Y)$. The process $Y(t)$ is separably valued because $\widetilde{X}(t)$ is, and $\int_{t}^{t+1}\|Y(u)\|_{2}^{p} d u<\infty$ for every $t$ because $\| \widetilde{X}(u+$ $f(u))\left\|_{2}^{p}=\right\| \widetilde{X}(0) \|_{2}^{p}$ from the stationarity of $\tilde{X}$. To complete the proof we will show that for every $\varepsilon>0$ there exists $\varepsilon^{\prime}>0$ such that if

$$
\sup _{t} \int_{t}^{t+1}\left|f\left(u+t^{\prime}\right)-f(u)\right|^{p} d u<\varepsilon^{\prime}
$$

then

$$
\sup _{t} \int_{t}^{t+1}\left\|\tilde{X}\left(f\left(u+t^{\prime}\right)\right)-\tilde{X}(f(u))\right\|_{2}^{p} d u<\varepsilon .
$$

Then taking $U\left(t^{\prime}\right)$ to be the shift group for the process $\tilde{X}$ we find

$$
\left\|Y\left(t+t^{\prime}\right)-U\left(t^{\prime}\right) Y(t)\right\|_{2}=\left\|\tilde{X}\left(f\left(t+t^{\prime}\right)\right)-\tilde{X}(f(t))\right\|_{2},
$$

and so for every $\varepsilon>0$

$$
S_{p}(\varepsilon, \tilde{X}, U) \supset S_{p}\left(\varepsilon^{\prime}, f\right)
$$

which will then imply that $S_{p}(\varepsilon, \widetilde{X}, U)$ is separable.

Thus let $\varepsilon>0$ be given. To prove the existence of $\varepsilon^{\prime}$ we first note that the continuity of the correlation of $\widetilde{X}$ implies that there exists $\delta>0$ such that if $u \in M_{\delta}$ where

$$
M_{\delta}=\left\{u \in[t, t+1]:\left|f\left(u+t^{\prime}\right)-f(u)\right|<\delta\right\}
$$

then

$$
\left\|\tilde{X}\left(f\left(u+t^{\prime}\right)\right)-\tilde{X}(f(u))\right\|_{2}^{p}<\frac{\varepsilon}{2} .
$$

Now

$$
\begin{aligned}
& \int_{t}^{t+1}\left\|\tilde{X}\left(f\left(u+t^{\prime}\right)\right)-\tilde{X}(f(u))\right\|_{2}^{p} d u \\
& \quad=\left(\int_{M_{\delta} \cap[t, t+1]}+\int_{[t, t+1] \backslash M_{\delta}}\right)\left\|\tilde{X}\left(f\left(u+t^{\prime}\right)\right)-\tilde{X}(f(u))\right\|_{2}^{p} d u \\
& \quad \leqslant \frac{\varepsilon}{2} m\left\{M_{\delta}\right\}+2 \sup _{u}\|\tilde{X}(u)\|_{2}^{p} m\left\{[t, t+1] \backslash M_{\delta}\right\}
\end{aligned}
$$


where $m$ denotes Lebesgue measure and $\sup _{u}\|\widetilde{X}(u)\|_{2}<\infty$ because $\tilde{X}$ is stationary. But $m\{[t, t+1]\}=1$ and by Chebyshev's inequality

$$
m\left\{[t, t+1] \backslash M_{\delta}\right\} \leqslant \frac{1}{\delta^{p}} \int_{t}^{t+1}\left|f\left(u+t^{\prime}\right)-f(u)\right|^{p} d u .
$$

It follows that

$$
\sup _{u} \int_{t}^{t+1}\left|f\left(u+t^{\prime}\right)-f(u)\right|^{p} d u<\varepsilon^{\prime}
$$

with $\varepsilon^{\prime}=(\varepsilon / 2) \delta^{p}\left(2 \sup _{u}\|\tilde{X}(u)\|_{2}^{p}\right)^{-1}$ implies

$$
\int_{t}^{t+1}\left\|\tilde{X}\left(f\left(u+t^{\prime}\right)\right)-\tilde{X}(f(u))\right\|_{2}^{p} d u<\varepsilon
$$

which proves the claim and hence the proposition.

It is known that $X(t)$ is APU in the Bohr sense if and only if $X(t)=$ $U(t)[P(t)]$ where $U(t)$ is a strongly continuous group of unitary operators on $H_{X}$ and $P(t)$ is a $L_{2}(\Omega)$-valued almost periodic function in the sense of Bohr. To approach a similar result in this case we must see if the conditions $A$ and $B$ are preserved under the action of the unitary group.

Proposition 3.2. If $\{U(t), t \in \mathbf{R}\}$ is a strongly continuous group of unitary operators on $H_{X}$ then

$$
Y(t)=U(t)[X(t)]
$$

satisfies conditions $\mathrm{A}$ and $\mathrm{B}$ if and only if $X(t)$ satisfies the same conditions.

$\mathrm{R}$ e $\mathrm{m}$ a $\mathrm{r} \mathrm{k}$. The sense of equality in (3.1) is in $L_{2}(\Omega)$ for almost every $t$, so that equality in the sense of the Stepanov norm:

$$
\|f\|_{S_{p}}=\sup _{t}\left(\int_{t}^{t+1}\|f(u)\|_{2}^{p} d u\right)^{1 / p}
$$

is implied.

P r o o f. We shall only give the sufficiency as $U^{-1}(t)=U(-t)$ is also strongly continuous group of unitary operators on $H_{Y}$. To show $Y(t)$ satisfies A we show it locally satisfies $A_{1}$ and $A_{2}$ where $X(t)$ satisfies $A_{1}$ and $A_{2}$. Since $X(t)$ is locally almost separably valued, then for any arbitrary $t$ it is separably valued for $s \notin E_{t} \subseteq(t, t+1)$ where $\operatorname{Leb}\left(E_{t}\right)=0$. Let $\left\{\xi_{j} ; j=\right.$ $1,2, \ldots\}$ be a CONS of $\overline{\operatorname{sp}}\left\{X(s), s \in(t, t+1), s \notin E_{t}\right\}$ so that for such $s$ we can write

$$
X(s)=\sum_{j=1}^{\infty}\left\langle X(s), \xi_{j}\right\rangle \xi_{j}
$$

where the coefficient functions $f_{j}(s)=\left\langle X(s), \xi_{j}\right\rangle$ are measurable because $X$ is weakly measurable $\left(A_{1}\right)$ and $\sum_{j=1}^{\infty}\left|f_{j}(s)\right|^{2}<\infty$ for $s \notin E_{t}$. For such $s$ we can write

$$
Y(s)=U(s)[X(s)]=\sum_{j=1}^{\infty}\left\langle X(s), \xi_{j}\right\rangle U(s)\left[\xi_{j}\right]
$$


because (3.2) is strongly convergent. But (3.3) shows that for $s \notin E_{t}$ the range of $Y$ is separable because the processes $a_{j}(s)=U(s)\left[\xi_{j}\right]$ are all continuous and thus separable; the scalar coefficients $f_{j}(s)=\left\langle X(s), \xi_{j}\right\rangle$ in (3.3) can only eliminate vectors. So $Y$ satisfies $\mathrm{A}_{2}$.

To show $Y$ satisfies $A_{1}$, we express

$$
\langle Y(s), Z\rangle=\sum_{j=1}^{\infty}\left\langle X(s), \xi_{j}\right\rangle\left\langle U(s)\left[\xi_{j}\right], Z\right\rangle=\sum_{j=1}^{\infty} f_{j}(s) g_{j}(s)
$$

where the $f_{j}$ are measurable, the $g_{j}$ are continuous and hence measurable, and the sum converges absolutely for $s \notin E_{t}$, and so $\langle Y(s), Z)$ is equal almost everywhere to a measurable function.

Finally, to show $\int_{t}^{t+1}\|Y(u)\|_{2}^{p} d u<\infty$ for every $t$ (B), we just note that $\|Y(u)\|_{2}^{p}=\|U(u)[X(u)]\|_{2}^{p}=\|X(u)\|_{2}^{p}$.

Corollary 3.1. If $X$ and $Y$ are related as in (3.1) by a strongly continuous group $\{U(t), t \in \mathbf{R}\}$ of unitary operators, then $Y$ is locally $L_{2}^{p}$ if and only if $X$ is locally $L_{2}^{p}$.

The following is the basic characterization of $S_{p} \mathrm{APU}$ processes. It is the $S_{p}$ version of Proposition 1 of [6].

Proposition 3.3. A second order stochastic process $X=X(t), t \in \mathbf{R}$, is $S_{p} \mathrm{APU}$ with a shift group $U$ if and only if

$$
X(t)=U(t)[P(t)]
$$

for some $S_{p}\left(H_{X}\right)$ almost periodic function $P(t)$.

$\mathrm{P} \mathrm{r}$ o of. In view of Proposition 3.2 we only need to show that $S_{p}(\varepsilon, X, U)$ is RD if and only if $S_{p}(\varepsilon, P)$ is RD where $P(t)=U(-t)[X(t)]$; the proof is almost identical to the proof of Theorem 1 of [6]. If $X(t)$ is given by (3.4) where $P(t)$ is an $S_{p}\left(H_{X}\right)$ AP function, then by Corollary $3.1 X$ is locally $L_{2}^{p}$. Further, in view of

$$
\int_{t}^{t+1}\left\|X\left(u+t^{\prime}\right)-U\left(t^{\prime}\right) X(u)\right\|_{2}^{p} d u=\int_{t}^{t+1} \cdot\left\|P\left(u+t^{\prime}\right)-P(u)\right\|_{2}^{p} d u
$$

we conclude $S_{p}(\varepsilon, X, U)=S_{p}(\varepsilon, P)$ is RD so $X$ is $S_{p}$ APU. Conversely, if $X$ is $S_{p}$ APU with the unitary group $U$ then $P(t)=U(-t) X(t)$ is locally $L_{2}^{p}$ and $p$-integrable and (3.5) again implies $S_{p}(\varepsilon, P)=S_{p}(\varepsilon, X, U)$ and hence $P$ is $S_{p}\left(H_{X}\right)$ almost periodic.

Some basic properties of $S_{p} \mathrm{APU}$ processes are summarized in the following proposition.

Theorem 3.1. Let $X(t)$ be an $S_{p} \mathrm{APU}$ process with the unitary group $U$. Then

(i) $X$ is $S_{p}$-bounded, i.e., $\sup _{t} \int_{t}^{t+1}\|X(u)\|_{2}^{p} d u<\infty$;

(ii) $X$ is $S_{p}$-uniformly continuous, i.e., $\lim _{h_{\rightarrow} \rightarrow 0} \sup _{t} \int_{t}^{t+1} \| X(u+h)-$ $X(u) \|_{2}^{p} d u=0$; 
(iii) $U$ is $S_{p}$-equicontinuous on $X$, i.e., $\lim _{h \rightarrow 0} \sup _{t} \int_{t}^{t+1} \| U(h) X(u)-$ $X(u) \|_{2}^{p} d u=0$

(iv) The function $Z(v, u)=U(v) P(u)=U(v) U(-u) X(u)$ is jointly $S_{p}$-continuous, i.e.,

$$
\lim _{h, k \rightarrow 0} \sup _{t, s} \int_{t}^{t+1} \int_{s}^{s+1}\|Z(v+k, u+h)-Z(v, u)\|_{2}^{p} d v d u=0 .
$$

P r o o f. From (3.1) we have

$$
\sup _{t} \int_{t}^{t+1}\|X(u)\|_{2}^{p} d u=\sup _{t} \int_{t}^{t+1}\|P(u)\|_{2}^{p} d u
$$

where $P$ is an $S_{p}\left(H_{X}\right)$ almost periodic function but for any such function the last expression is finite (see, e.g., $[9$, p. 34]). This proves (i).

To prove (ii) observe that since $X$ is locally $L_{2}^{p}$ integrable, for any $t$ it is continuous in the sense

$$
\lim _{h \rightarrow \infty} \int_{t}^{t+1}\|X(u+h)-X(u)\|_{2}^{p} d u=0
$$

and so it will be uniformly continuous in this sense for $t$ in any bounded interval $(a, b)$. As in the case of UAP functions, the question of continuity at an arbitrary $t$ can be translated to a single bounded interval. Thus for $\varepsilon>0$ and arbitrary $t$ write $t=t^{\prime}+t^{\prime \prime}$. where $t^{\prime \prime} \in\left[0, l_{\varepsilon}\right]$ and $t^{\prime} \in S(\varepsilon, X)$. Performing obvious changes of variables and adding and subtracting $U\left(t^{\prime}\right) X(u+t)$ and $U\left(t^{\prime}\right) X(u)$ we get

$$
\begin{aligned}
& \left(\int_{t}^{t+1}\|X(u+h)-X(u)\|_{2}^{p} d u\right)^{1 / p} \\
& \quad=\left(\int_{t^{\prime \prime}}^{t^{\prime \prime}+1}\left\|X\left(u+t^{\prime}+h\right)-X\left(u+t^{\prime}\right)\right\|_{2}^{p} d u\right)^{1 / p} \leqslant \varepsilon_{1}+\varepsilon_{2}+\varepsilon_{3}
\end{aligned}
$$

where

$$
\begin{aligned}
\varepsilon_{1}^{p} & =\int_{t^{\prime \prime}}^{t^{\prime \prime}+1}\left\|X\left(u+t^{\prime}+h\right)-U\left(t^{\prime}\right) X(u+h)\right\|_{2}^{p} d u, \\
\varepsilon_{2}^{p} & =\int_{t^{\prime \prime}}^{t^{\prime \prime}+1}\left\|U\left(t^{\prime}\right) X(u+h)-U\left(t^{\prime}\right) X(u)\right\|_{2}^{p} d u, \\
\varepsilon_{3}^{p} & =\int_{t^{\prime \prime}}^{t^{\prime \prime}+1}\left\|U\left(t^{\prime}\right) X(u)-X\left(u+t^{\prime}\right)\right\|_{2}^{p} d u .
\end{aligned}
$$

Now since $t^{\prime} \in S(\varepsilon, X), \varepsilon_{1}^{p}$ and $\varepsilon_{3}^{p}$ are bounded by $\varepsilon$. By taking $|h|$ sufficiently small, the remaining term $\varepsilon_{2}^{p}$ is no bigger than $\varepsilon$ from the uniform continuity of $X$ (in the sense of (3.2)) on any bounded open interval containing $\left[0, l_{\varepsilon}\right]$. 
Turning to (iii), write

$$
\begin{aligned}
& \sup _{t}\left(\int_{t}^{t+1}\|U(h) X(u)-X(u)\|_{2}^{p} d u\right)^{1 / p} \\
& \leqslant \sup _{t}\left(\int_{t}^{t+1}\|U(h) X(u)-X(u+h)\|_{2}^{p} d u\right)^{1 / p} \\
& \quad+\sup _{t}\left(\int_{t}^{t+1}\|X(u+h)-X(u)\|_{2}^{p} d u\right)^{1 / p} .
\end{aligned}
$$

The second term goes to zero as $h \rightarrow 0$ by (ii). The first one equals

$$
\sup _{t}\left(\int_{t}^{t+1}\|P(u+h)-P(u)\|_{2}^{p} d u\right)^{1 / p}
$$

where $P$ is the $S_{p}\left(H_{X}\right)$ AP function from Proposition 3.3. It converges to zero by the uniform $S_{p}$ continuity of $S_{p}$ almost periodic functions $([9, \mathrm{p} .33])$. Finally (iv) follows by noting that

$$
\begin{aligned}
\sup _{t, s} & \left(\int_{s}^{s+1} \int_{t}^{t+1}\|Z(v+k, u+h)-Z(v, u)\|_{2}^{p} d u d r\right)^{1 / p} \\
\leqslant & \sup _{t, s}\left(\int_{s}^{s+1} \int_{t}^{t+1}\|(U(v+k)-U(v)) P(u+h)\|_{2}^{p} d u d v\right)^{1 / p} \\
& +\sup _{t, s}\left(\int_{s}^{s+1} \int_{t}^{t+1}\|U(v) P(u+h)-U(v) P(u)\|_{2}^{p} d u d v\right)^{1 / p}
\end{aligned}
$$

and applying (iii) to the first term and using the $S_{p}$ continuity of $p$ in the second.

We next give representations of $S_{p} \mathrm{APU}$ processes, analogous to those given in [6] for uniformly APU processes, based on $X(t)=U(t)[P(t)]$ where the sense of equality is that of the Stepanov norm. The first is based on Stone's theorem on representation of unitary groups [11] which in our case gives

$$
X(t)=U(t) P(t)=\int_{-\infty}^{+\infty} \exp (i \gamma t) d E_{\gamma}[P(t)] .
$$

The second is based on a representation of an $S_{p} \mathrm{AP}$ function as a limit in Stepanov norm of a sequence of trigonometric polynomials. The third is based on expression (3.2) in the proof of Proposition 3.2. The proofs of the first two are identical to those in [6] and are therefore omitted.

Proposition 3.4. A process $X=X(t), t \in \mathbf{R}$, is $S_{p} \mathrm{APU}$ if and only if it has a representation

$$
X(t)=\int_{-\infty}^{+\infty} \exp (i \gamma t) Z(d \gamma, t)
$$


where for each $t, Z(\cdot, t)$ is an $L_{2}(\Omega)$-valued measure orthogonally scattered in that for every $t, s$ and disjoint $A, B \in Z(A, t) Z(B, s)=0$ and for each $A, Z(A, \cdot)$ is $S_{p}\left(H_{X}\right)$ almost periodic.

Proposition 3.5. A process $X=X(t), t \in \mathbf{R}$, is $S_{p} \mathrm{APU}$ if and only if it is an $S_{p}$-limit of processes of the form $X_{n}(t)=\sum_{j=1}^{k_{n}} \eta_{j, n}(t) \exp \left\{i \gamma_{j} t\right\}$ where $\left\{\eta_{j, n}\right\}$ is a set of jointly wide sense stationary stochastic processes and $\left\{\gamma_{j}\right\}$ is a countable set of real numbers.

Theorem 3.2. The process $X=X(t), t \in \mathbf{R}$, is $S_{p} \mathrm{APU}$ if and only if

$$
X(t)=\sum_{j=1}^{\infty} f_{j}(t) a_{j}(t)
$$

where the sequence $\left\{a_{j}(t), j \in \mathbf{Z}\right\}$ consists of jointly stationary processes with $\mathbf{E}\left\{\left|a_{j}(0)\right|^{2}\right\}=1 \forall j$ that are mutually instantaneously orthogonal, $\mathbf{E}\left\{a_{j}(t) \overline{a_{k}(t)}\right\}=0$ for every $t$ and $j \neq k$. The sequence $\left\{f_{j}(t), j \in \mathbf{Z}\right\}$ consists of scalar $S_{p}$ functions for which $\lim _{N \rightarrow \infty} \int_{t}^{t+1} \sum_{j>N}\left|f_{j}(s)\right|^{2} d s=0$ uniformly in $t$.

$\mathrm{P}$ r o of. Necessity. If $X$ is $S_{p} \mathrm{APU}$ it is locally almost separably valued; that is, for every $k \in \mathbf{Z}, \overline{\operatorname{sp}}\left\{X(s), s \in[k, k+1) \backslash E_{k}\right\}$ is separable where $\operatorname{Leb}\left(E_{k}\right)=0$. It follows that $\overline{\mathrm{sp}}\left\{X(s), s \notin \cup_{k} E_{k}\right\}$ is separable. Let $\left\{\varepsilon_{j} ; j=1,2, \ldots\right\}$ be a CONS of $\overline{\operatorname{sp}}\left\{X(s), s \notin \cup_{k} E_{k}\right\}$ so that for a.e. $s$ we can write

$$
P(s)=\sum_{j=1}^{\infty}\left\langle P(s), \xi_{j}\right\rangle_{2} \xi_{j}
$$

where the coefficient functions $f_{j}(s)=\left\langle P(s), \xi_{j}\right\rangle_{2}$ are measurable because $X$ is weakly measurable, and are locally $L^{p}$ because $\left|f_{j}(s)\right|=\left|\left\langle P(s), \xi_{j}\right\rangle_{2}\right| \leqslant$ $\|P(s)\|_{2}$. Each $f_{j}(s)$ is a scalar $S_{p}$ function because

$$
\left|f_{j}\left(s+t^{\prime}\right)-f_{j}(s)\right|=\left|\left\langle P\left(s+t^{\prime}\right)-P(s), \xi_{j}^{t}\right\rangle_{2}\right| \leqslant\left\|P\left(s+t^{\prime}\right)-P(s)\right\|_{2} .
$$

Using $X(t)=U(t)[P(t)]$ we identify $a_{j}(t)=U(t) \xi_{j}$, and note that $\left\langle a_{j}(t)\right.$, $\left.a_{k}(t)\right\rangle_{2}=\left\langle U(t) \xi_{j}, U(t) \xi_{k}\right\rangle_{2}=\left\langle\xi_{j}, \xi_{k}\right\rangle_{2}=\delta_{j k}$.

To show $\int_{t}^{t+1} \sum_{j>N}\left|f_{j}(s)\right|^{2} d s \longrightarrow 0$ uniformly in $t$ as $N \rightarrow \infty$, we first observe that

$$
\begin{aligned}
\int_{t}^{t+1}\left\|X(u)-X_{N}(u)\right\|_{2}^{2} d u & =\int_{t}^{t+1}\left\|P(u)-P_{N}(u)\right\|_{2}^{2} d u \\
& =\int_{t}^{t+1} \sum_{j \geqslant N}\left|f_{j}(u)\right|^{2} d u
\end{aligned}
$$

where $P_{N}(t)=\sum_{j \leqslant N} f_{j}(t) \xi_{j}$ and $X_{N}(t)=U(t)\left[P_{N}(t)\right]$. We next adapt a proof of Levitan $\left(\left[9\right.\right.$, p. 75]) to show that $P_{N}(t)$ converges in the Stepanov norm uniformly to $P(t)$, and with the help of $(3.10)$, the result will be concluded. 
If $P(t)$ is $S\left(H_{X}\right)$ AP, then for every $t$, the function $\widetilde{P}_{t}$ defined by $\widetilde{P}_{t}(s)=$ $P(t+s)$ is a map from $\mathbf{R}$ to the locally measurable functions $\left\{X: \int_{0}^{1} \| X(t+\right.$ $u) \|_{2}^{2} d u<\infty$ for every $\left.t\right\}$. An inner product may be defined on these functions by $\langle X, Y\rangle=\int_{0}^{1}\langle X(u), Y(u)\rangle_{2} d u$, and since we already know they form a Banach space, they evidently also are a Hilbert space which we denote as $\mathcal{H}$. Now let $\mathcal{H}\left(\widetilde{P}_{t} ; t \in \mathbf{R}\right)$ be the subspace of $\mathcal{H}$ generated by the uniformly almost periodic function $\widetilde{P}_{t}$; since $\widetilde{P}: \mathbf{R} \rightarrow \mathcal{H}$ is continuous, it follows that $\mathcal{H}\left(\widetilde{P}_{t} ; t \in \mathbf{R}\right)$ is separable, and we take $\left\{e_{\mu}, \mu \in \mathbf{Z}\right\}$ to be a CONS for $\mathcal{H}\left(\widetilde{P}_{t} ; t \in \mathbf{R}\right)$. Expect for a set of Lebesgue measure zero, every $e_{\mu}$ has a representation

$$
e_{\mu}(s)=\sum_{j=1}^{\infty}\left\langle e_{\mu}(s), \xi_{j}\right\rangle_{2} \xi_{j}
$$

where for those $s$

$$
\sum_{j=1}^{\infty}\left|\left\langle e_{\mu}(s), \xi_{j}\right\rangle_{2}\right|^{2}<\infty
$$

From the argument subsequent to (3.9) it follows that the complex valued functions $\left\langle e_{\mu}(s), \xi_{j}\right\rangle_{2}$ are $L_{2}[0,1]$ and

$$
\left\|\left.\left|e_{\mu}\left\|_{2}^{2}=\int_{0}^{1}\right\| e_{\mu}(s) \|_{2}^{2} d u \int_{0}^{1} \sum_{j=1}^{\infty}\right|\left\langle e_{\mu}(s), \xi_{j}\right\rangle_{2}\right|^{2} d s=1 .\right.
$$

If $\left\{\varphi_{j}, j \in \mathbf{N}^{+}\right\}$is a CONS for $L_{2}[0,1]$, then we may conclude that another CONS for $\mathcal{H}\left(\widetilde{P}_{t} ; t \in \mathbf{R}\right)$ may be taken from the functions $\left\{\varphi_{m}(s) \xi_{n}, m \in\right.$ $\left.\mathbf{N}^{+}, n \in \mathbf{N}^{+}\right\}$. Denote this new CONS by $\Psi=\left\{\psi_{m}, m \in \mathbf{N}^{+}\right\}$.

We are now ready to show the uniform convergence of (3.11). Since the function $\widetilde{P}_{t}$ is UAP, the closure of its range $\overline{\mathcal{R}}\left(\widetilde{P}_{t} ; t \in \mathbf{R}\right)$ is a compact subset of the Hilbert space $\mathcal{H}\left(\widetilde{P}_{t} ; t \in \mathbf{R}\right.$ ) (which is thus a complete metric space). Hence $\overline{\mathcal{R}}\left(\widetilde{P}_{t} ; t \in \mathbf{R}\right)$ is totally bounded, and so for any $\varepsilon>0$ there is a finite collection of vectors $\left\{\eta_{1}, \eta_{2}, \ldots, \eta_{n}\right\} \subset \mathcal{H}\left(\widetilde{P}_{t} ; t \in \mathbf{R}\right)$ such that for every fixed $t$ one of these vectors yields $\left\|\widetilde{P}_{t}-\eta_{k}\right\|_{2}<\varepsilon / 2$; and each $\eta_{k}$ has a finite approximant $\widehat{\eta}_{k}=\sum_{j=1}^{N_{k}} \alpha_{j k} \psi_{j}$ for which $\left\|\widehat{\eta}_{k}-\eta_{k}\right\|_{2}<\varepsilon / 2$. This leads to the conclusion that for every $t$ there is an approximant $\widehat{\eta}_{k}$ for which

$$
\left\|\widetilde{P}_{t}-\widehat{\eta}_{k}\right\|_{2}<\varepsilon
$$

that is, there exists a finite collection from $\Psi$, linear combinations of which uniformly approximate $\widetilde{P}_{t}$. Let $N$ be the largest index of the $\xi_{n}$ appearing in all the approximants $\widehat{\eta}_{k}$ and let $S_{N}$ be the subspace of $\mathcal{H}\left(\widetilde{P}_{t} ; t \in \mathbf{R}\right)$ formed by functions of the form $\sum_{j \leqslant N} g_{j}(t) \xi_{j}$ where $g_{j} \in L_{2}[0,1]$. Then it is clear that all the approximants $\widehat{\eta}_{k} \in S_{n}$, and additionally $\widetilde{P}_{N, t}=\sum_{j \leqslant N}\langle P(t+$ $\left.s), \xi_{j}\right\rangle_{2} \xi_{j} \in S_{N}$. But $\widetilde{P}_{N, t}$ is just the projection of $\widetilde{P}_{t}$ onto $S_{N}$ and hence (3.13) implies that $\left\|\left|\widetilde{P}_{t}-\widetilde{P}_{N, t}\right|\right\|<\varepsilon$ for all $t$. 
Sufficiency. First, the joint stationarity of $\left\{a_{j}(t), j \in \mathbf{Z}\right\}$ implies there is a. unitary group $\{U(\tau), \tau \in \mathbf{R}\}$ which, for every $j$, gives $a_{j}(t)=U(t) \xi_{j}$ where $\xi_{j}=a_{j}(0)$; the instantaneous orthogonality gives $\left\langle\xi_{j}, \xi_{k}\right\rangle=\delta_{j k}$. We now wish to show that $P: \mathbf{R} \rightarrow H_{X}$ given by $P(t)=\sum_{j} f_{j}(t) \xi_{j}$ is $S_{p}\left(H_{X}\right) \mathrm{AP}$, where $\left\{f_{j}(t)\right\}$ are scalar $S_{p}$ functions that satisfy $\int_{t}^{t+1} \sum_{j>N}\left|f_{j}(s)\right|^{2} d s \longrightarrow 0$ uniformly in $t$ as $N \rightarrow \infty$. But from (3.11) this is enough to conclude that $P$ is locally $L_{2}^{2}$ and that $P_{N}(t)=\sum_{j \leqslant N} f_{j}(t) \xi_{j}$ converges in the Stepanov norm to $P(t)$ and thus $P(t)$ must be $S_{p}\left(H_{X}\right)$ AP.

\section{Harmonic analysis of the correlation for $S_{p} \mathrm{APU}$ processes}

We note that the Bohr APU processes may be shown to be uniformly $\mathrm{APC}$ in the sense of Dehay [2], and also equi-APC as well. In the current case, we show only that $S_{2 p} \mathrm{APU}$ processes are equi- $S_{p} \mathrm{APC}$.

Proposition 4.1. If $X=X(t), t \in \mathbf{R}$, is $S_{2 p} \mathrm{APU}$, then the family of functions $\{B(\cdot, \tau)=R(\cdot+\tau, \cdot), \tau \in \mathbf{R}\}$ is equi- $S_{p} \mathrm{AP}$.

P r o of. First we must show that $B(t, \tau)$ is locally $L_{p}: \int_{0}^{1} \mid B(t+$ $u, \tau)\left.\right|^{p} d u<\infty$ for every $t$. This follows immediately from an application of the Schwarz inequality (on $\left.L_{2}[0,1]\right)$ to the function $|B(t+u, \tau)|^{p} \leqslant$ $\|X(t+u+\tau)\|^{p}\|X(t+u)\|^{p}$. For fixed $\tau$ we replace $B(\cdot, \tau)$ by a measurable equivalent, as required; condition $\mathrm{B}$ implies that $\|X(t+\cdot)\|^{p}$ is Lebesgue measurable. To complete the result, we must show that

$$
S(B, \varepsilon)=\left\{t^{\prime}: \sup _{\tau \in \mathbf{R}} \sup _{t \in \mathbf{R}} \int_{0}^{1}\left|B\left(t+u+t^{\prime}, \tau\right)-B(t+u, \tau)\right|^{p} d u<\varepsilon\right\}
$$

is relatively dense. For fixed $t$, write

$$
X\left(t+t^{\prime}+\tau\right)=U\left(t^{\prime}\right) X(t+\tau)+\varepsilon\left(t+\tau, t^{\prime}\right)
$$

where $U(\cdot)$ is a shift group for $X$, and $\left\{t^{\prime}: \sup _{t \in \mathbf{R}} \sup _{t \in \mathbf{R}} \int_{0}^{1} \| \varepsilon(t+u+\right.$ $\left.\left.\tau, t^{\prime}\right) \|^{2 p} d u<\varepsilon\right\}$ is RD. Then using (4.1) produces

$$
\begin{aligned}
& R\left(t+u+t^{\prime}+\tau, t+u+t^{\prime}\right)-R(t+u+\tau, t+u) \\
& \quad=\left\langle U\left(t^{\prime}\right) X(t+u+\tau), \varepsilon\left(t+u, t^{\prime}\right)\right\rangle+\left\langle U\left(t^{\prime}\right) X(t+u), \varepsilon\left(t+u+\tau, t^{\prime}\right)\right\rangle \\
& \quad+\left\langle\varepsilon\left(t+u+\tau, t^{\prime}\right), \varepsilon\left(t+u, t^{\prime}\right)\right\rangle .
\end{aligned}
$$

Application of the triangle inequality to $\mathbf{C}$ and then the Schwarz inequality (in $L_{2}(\Omega)$ ) to each term on the right gives the inequality

$$
\begin{aligned}
& \left|B\left(t+u+t^{\prime}, \tau\right)-B(t+u, \tau)\right| \leqslant\left\|U\left(t^{\prime}\right) X(t+u+\tau)\right\|\left\|\varepsilon\left(t+u, t^{\prime}\right)\right\| \\
& \quad+\left\|U\left(t^{\prime}\right) X(t+u)\right\|\left\|\varepsilon\left(t+u+\tau, t^{\prime}\right)\right\|+\left\|\varepsilon\left(t+u+\tau, t^{\prime}\right)\right\|\left\|\varepsilon\left(t+u, t^{\prime}\right)\right\| .
\end{aligned}
$$

We next apply the triangle inequality on $L_{p}[0,1]$, the unitarity of $U(\cdot)$ and then the Schwarz inequality on $L_{2}[0, T]$ to obtain bounding terms of the form 
(taking the first on the right of the inequality)

$$
\left[\int_{0}^{1}\|X(t+u+\tau)\|^{2 p} d u\right]^{1 /(2 p)}\left[\int_{0}^{1}\left\|\varepsilon\left(t+u, t^{\prime}\right)\right\|^{2 p} d u\right]^{1 /(2 p)} .
$$

Taking the supremum over $t$ and $\tau$, the first is dominated (say by $M_{X}$ ) and the second is no larger than $\left[\varepsilon_{0}\right]^{1 /(2 p)}$ provided $t^{\prime} \in S_{p}\left(\varepsilon_{0}, X, U\right)$. Combining all the terms produces

$$
\begin{aligned}
& \sup _{\tau \in \mathbf{R}} \sup _{t \in \mathbf{R}} \int_{0}^{1}\left|B\left(t+u+t^{\prime}, \tau\right)-B(t+u, \tau)\right|^{p} d u \\
& \quad \leqslant 2 M_{X}\left[\varepsilon_{0}\right]^{1 /(2 p)}+\left[\varepsilon_{0}\right]^{1 / p} \equiv K\left(\varepsilon_{0}\right)
\end{aligned}
$$

provided $t^{\prime} \in S_{p}\left(\varepsilon_{0}, X, U\right)$. Since $K\left(\varepsilon_{0}\right)$ is monotone for $\varepsilon_{0} \geqslant 0$, we have for any $\varepsilon>0$,

$$
S(B, \varepsilon) \supset S_{p}\left(K^{-1}(\varepsilon), X, U\right) .
$$

Recall that

$$
a(\lambda, \tau)=\lim _{n \rightarrow \infty} \frac{1}{2 T} \int_{-T}^{T} R(t+\tau, t) \exp (-i \lambda t) d t
$$

exists for every $\lambda$ and $\tau$ and determines $B(t, \tau)$ uniquely almost everywhere (in the sense described in Section 2). For every $\tau$ the set $\Lambda_{\tau}=\{\lambda: a(\lambda, \tau) \neq$ $0\}$ is countable; moreover, as we mentioned in the remark just before Theorem 2.1 , the continuity in $S_{p}$ norm implies that there exists one countable set $\Lambda=\left\{\lambda_{1}, \lambda_{2}, \ldots\right\}$ such that $a(\lambda, \tau)=0$ for $\lambda \notin \Lambda$ and every $\tau$. Thus $R(t+\tau, t)$ is uniquely (up to equality almost everywhere) represented by a countable sequence of Fourier frequencies $\left\{\lambda_{k}\right\}$ and coefficients $a\left(\lambda_{k}, \tau\right)$. We express this as in [9] by writing $R(t+\tau, t) \sim \sum_{k} a\left(\lambda_{k}, \tau\right) \exp \left(i \lambda_{k} t\right)$. The series on the right may not converge but there exists a sequence of multipliers $\mu_{k, n}$, $k=1,2, \ldots, n, n=1,2, \ldots$, such that for every $\tau$ the sequence

$$
R_{n}(t+\tau, t)=\sum_{k=1}^{n} \mu_{n, k} a\left(\lambda_{k}, \tau\right) \exp \left(i \lambda_{k} t\right)
$$

converges to $R(t+\tau, t)$ in Stepanov norm $([9$, p. 35]), i.e.,

$$
\lim _{n \rightarrow \infty} \sup _{t} \int_{t}^{t+1}\left|R(u+\tau, u)-R_{n}(u+\tau, u)\right|^{p} d u=0 .
$$

Due to their construction from the Fejer polynomials [9] the multipliers $\mu_{k, n}$ depend only of the frequencies $\left\{\lambda_{k}\right\}$ and are independent of $\tau$ and the coefficients $\left\{a\left(\lambda_{k}, \tau\right)\right\}$.

The following shows that the inherent continuity of a $S_{2}$ APU process is enough to ensure $B(\cdot, \tau)$ is continuous with respect to $\tau$ in the scalar $S_{2}$ sense, and by Theorem $2.2 a(\lambda, \tau)$, for each fixed $\lambda$, is a Fourier transform of a signed measure of finite total variation. 
Theorem 4.1. Let $X=X(t), t \in \mathbf{R}$, be $S_{2} \mathrm{APU}$. Then for every $\lambda \in \mathbf{R}$ the function $a(\lambda, \cdot)$ is a Fourier transform of a signed measure of finite total variation.

P r o o f. To apply Theorem 2.2 we need to show the required continuity. To do this we note

$$
\begin{aligned}
\sup _{t} & \left(\int_{t}^{t+1}|B(u, \tau+h)-B(u, \tau)|^{2} d u\right) \\
= & \sup _{t}\left(\int_{t}^{t+1}|\mathbf{E}\{X(u+\tau+h) \bar{X}(u)\}-\mathbf{E}\{X(u+\tau) \overline{X(u)}\}|^{2} d u\right) \\
\leqslant & \sup _{t}\left(\int_{t}^{t+1}\|X(u+\tau+h)-X(u+\tau)\|_{2}^{2} d u\right)^{1 / 2} \\
& \times \sup _{t}\left(\int_{t}^{t+1}\|X(u)\|_{2}^{2} d u\right)^{1 / 2} .
\end{aligned}
$$

By Theorem 3.1 the first term tends to zero as $h \rightarrow 0$ while the second term is bounded, thus proving the required continuity.

\section{REFERENCES}

1. Bochner S. Abstrakte Fastperiodische Functionen. - Acta Math., 1933, v. 61, p. 149184.

2. Dehay $D$. Nonlinear analysis for almost periodically correlated strongly harmonizable processes. - Preprint, 1990.

3. Folland G. B. Real Analysis. New York: Wiley-Interscience, 1984.

4. Гладыше $E$. Г. Периодически и почти-периодически коррелированные случайные процессы с непрерывным временем. - Теория вероятн. и ее примен., 1963, т. VIII, в. 2 , с. 173-177.

5. Hurd H. L. Periodically correlated processes with discontinuous correlation functions. - Теория вероятн. и ее примен., 1974, т. XIX, в. 4, с. 834-838.

6. Hurd H. L. Almost periodically unitary stochastic processes. - Stoch. Process. Appl., 1992 , v. 43, p. 99-113.

7. Hurd $H$. L. Correlation theory for almost periodically correlated processes. - J. Multivar. Anal., 1991, v. 37, № 1, p. 24-45.

8. Hurd H. L., Kallianpur G. Periodically correlated and periodically unitary processes and their relationship to $L_{2}[0, T]$-valued stationary sequences. - In: Nonstationary Stochastic Processes and Their Application. / Ed. by J. C. Hardin and A. G. Miamee. World Scientific Publishing, 1992.

9. Levitan B. M., Zhivkov V. V. Almost Periodic Functions and Differential Equations. Cambridge: Cambridge Univ. Press, 1982.

10. Stepanov $V$. V. Uber einige Verallgemeinerungen der fast periodischen Funktionen. Math. Ann., 1926, B. 95, S. 437-498.

11. Stone $M$. On one parameter unitary groups in Hilbert space. - Math. Ann., 1932, v. 33, № 2, p. 643-648.

12. Yosida K. Functional Analysis. New York: Springer-Verlag, 1970. 